\title{
Causes, Features, and Outcomes of Drug-Induced Liver Injury in 69 Children from China
}

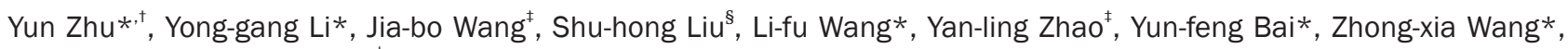
Jian-yu Li*, and Xiao-he Xiao ${ }^{\ddagger}$

${ }^{*}$ Integrative Medicine Center, 302 Military Hospital, ${ }^{+}$Medical School of Chinese PLA, ${ }^{\neq}$China Military Institute of Chinese Medicine and ${ }^{s}$ Department of Pathology, 302 Military Hospital, Beijing, China

Background/Aims: Drug-induced liver injury (DILI) is a frequent cause of pediatric liver disease; however, the data on DILI are remarkably limited. Methods: All 69 children hospitalized with DILI between January 2009 and December 2011 were retrospectively studied. Results: A total of $37.7 \%$ of the children had medical histories of respiratory infection. The clinical injury patterns were as follows: hepatocellular $89.9 \%$, cholestatic $2.9 \%$, and mixed $7.2 \%$. Liver biopsies from 55 children most frequently demonstrated chronic (47.3\%) and acute (27.3\%) hepatitis. Hypersensitivity features, namely, fever (31.9\%), rash (21.7\%), and eosinophilia (1.4\%), were found. Twenty-four children (34.8\%) developed chronic DILI. Antibiotics (26.1\%) were the most common Western medicines (WMs) causing DILI, and the major implicated herbs were Ephedra sinica and Polygonum multiflorum. Compared with WM, the children whose injuries were caused by Chinese herbal medicine ( $\mathrm{CHM}$ ) showed a higher level of total bilirubin ( $1.4 \mathrm{mg} / \mathrm{dL}$ vs $16.6 \mathrm{mg} / \mathrm{dL}, \mathrm{p}=0.004)$ and a longer prothrombin time ( 11.8 seconds vs 17.3 seconds, $p=0.012$ ), but they exhibited less chronic DILI (2/15 vs $18 / 39$, $\mathrm{p}=0.031$ ). Conclusions: Most cases of DILI in children are caused by antibiotics or CHM used to treat respiratory infection and present with hepatocellular injury. Compared with WM, CHM is more likely to cause severe liver injury, but liver injury caused by CHM is curable. (Gut Liver 2015;9:525533)

Key Words: Pediatric; Hepatotoxicity; Diagnosis; Chinese herbal medicine; Liver biopsy

\section{INTRODUCTION}

Drug-induced liver injury (DILI) is one of the most common reasons for pediatric liver disease and has become an important area of concern for clinicians, regulatory agencies, and pharmaceutical companies. In a retrospective research from China, 64 pediatric cases of DILI accounted for 10\% of 641 cases of children hospitalized with liver injury over a period of 5 years. ${ }^{1}$ Drug-induced acute liver failure (ALF) has been reported to be the main cause of ALF in children in the United States, Canada, and the United Kingdom. ${ }^{2,3}$

Systematic studies on children with DILI are scarce compared to the increasing number of reports on DILI in adults across all regions of the world. In the last decade, DILI in children has been described in case reports or small series. ${ }^{4-7} \mathrm{~A}$ recent prospective study over more than 5 years from Drug-Induced Liver Injury Network (DILIN) has reported the clinical and pathological characteristics of 30 children with DILI. ${ }^{8}$ Compared with adults, drug metabolism in children differs in terms of absorption, distribution, metabolism, and excretion. ${ }^{9,10}$ For example, the level of cytochromes P450, a class of enzymes responsible for metabolizing drugs, increases with age. ${ }^{11}$ Because of the scarcity of studies on DILI in children and age-related differences in drug metabolism, a National Institutes of Health clinical research workshop in 2008 concluded that additional studies of pediatric DILI were needed. ${ }^{12}$

Recently herbal therapy is increasingly being used in pediatric populations. In the United States, an estimated 2.9 million children and adolescents used herbs or dietary supplements according to the 2007 National Health Interview Survey. ${ }^{13}$ With the historical background of the use of Chinese herbal medicine (CHM), CHM is an important cause of DILI in China in both chil-

Correspondence to: Xiao-he Xiao

China Military Institute of Chinese Medicine, 302 Military Hospital, No. 100, the 4th West Ring Road, Beijing 10039, China

Tel: +86-10-6693-3322, Fax: +86-10-6693-3322, E-mail: pharmacy302@126.com

Received on May 11, 2014. Revised on July 21, 2014. Accepted on August 21, 2014. Published online February 26, 2015

pISSN 1976-2283 eISSN 2005-1212 http://dx.doi.org/10.5009/gnl14184

@. This is an Open Access article distributed under the terms of the Creative Commons Attribution Non-Commercial License (http://creativecommons.org/licenses/by-nc/4.0) which permits unrestricted non-commercial use, distribution, and reproduction in any medium, provided the original work is properly cited. 
dren and adults. ${ }^{6,14}$ Despite the worldwide application of herbal products, data regarding herbal hepatotoxicity in children and adolescents are remarkably limited. Thus, it is imperative to recognize and investigate herbal hepatotoxicity in children. ${ }^{15}$

Therefore, we undertook this study to analyze the causes, clinical, laboratory, and pathological features, and outcomes of DILI in children up to 14 years of age and compare the differences between CHM and Western medicine (WM) as implicated agents of pediatric liver injury.

\section{MATERIALS AND METHODS}

We retrospectively collected and analyzed all children up to 14 years of age hospitalized with DILI between January 2009 and December 2011 in the 302 Hospital of PLA, Beijing, China. Children were considered to have DILI if they met the following criteria: ${ }^{16-18}$ a clinical suspicion of drug-induced hepatotoxicity, as defined as recent onset abnormalities in liver tests, such as rise in serum total bilirubin (TB) of at least $2 \mathrm{mg} / \mathrm{dL}$, and/or alanine aminotransferase (ALT) or aspartate aminotransferase (AST) $>3$ times the upper limit of normal (ULN), or rise in alkaline phosphatase (ALP) $>2$ times the ULN with any rise in TB or ALT or AST; exclusion of viral hepatitis A to E, hepatitis caused by nonhepatotropic virus, autoimmune liver disease, hepatolenticular degeneration, and other causes of liver diseases; and based on the Roussel Uclaf Causality Assessment Method (RUCAM), highly probable (>8), probable (6 to 8), or possible (3 to 5) are considered drug-induced liver injury. The diagnosis of drug-induced liver injury in children is shown in Fig. 1. Demographic, clinical, laboratory, and pathological data of children with DILI were extracted from hospital records.

According to the Council for International Organizations of
Medical Sciences (CIOMS) scale, DILI is classified into either hepatocellular, cholestatic, or mixed patterns on the ratio of ALT (as a multiple of its ULN) to ALP (as a multiple of its ULN), designated as the $\mathrm{R}$ (for ratio) value. ${ }^{19}$ Hepatocellular DILI is defined as $R \geq 5$, cholestatic as $R \leq 2$, and mixed as $R>2$ to $R<5$. A diagnosis of drug-induced ALF was made from established criteria: ${ }^{2}$ children with no known evidence of chronic liver disease, biochemical evidence of acute liver injury, and hepatic-based coagulopathy defined as a prothrombin time (PT) $\geq 15$ seconds not corrected by Vitamin $\mathrm{K}$ in the presence of clinical hepatic encephalopathy or a PT $\geq 20$ seconds regardless of the presence or absence of clinical hepatic encephalopathy. Based on established guidelines, Chronic DILI is considered as persistent biochemical abnormalities 3 months after drug discontinuation for cases of hepatocellular DILI, and persistent abnormalities for 6 months for cases of cholestatic/mixed DILI. ${ }^{19}$

Liver biopsies were reviewed by two hepatopathologists who were blinded to clinical and demographic information. According to standard criteria, the pathological pattern of injury was categorized into acute hepatitis (predominantly lobular inflammation, with or without confluent or bridging necrosis; absence of cholestasis), chronic hepatitis (portal inflammation with interface hepatitis, with or without portal-based fibrosis; no cholestasis), acute cholestasis (hepatocellular and/or canalicular cholestasis; minimal inflammation), chronic cholestasis (duct sclerosis and loss; periportal cholate stasis; portal-based fibrosis; copper accumulation), cholestatic hepatitis (acute or chronic hepatitis pattern plus cholestasis) and other patterns. ${ }^{20,21}$ Individual histologic features were also recorded.

Simple descriptive statistics including medians, 25th to 75th percentiles, frequencies, and percentages were used to summarize the data. Continuous variables were compared using the

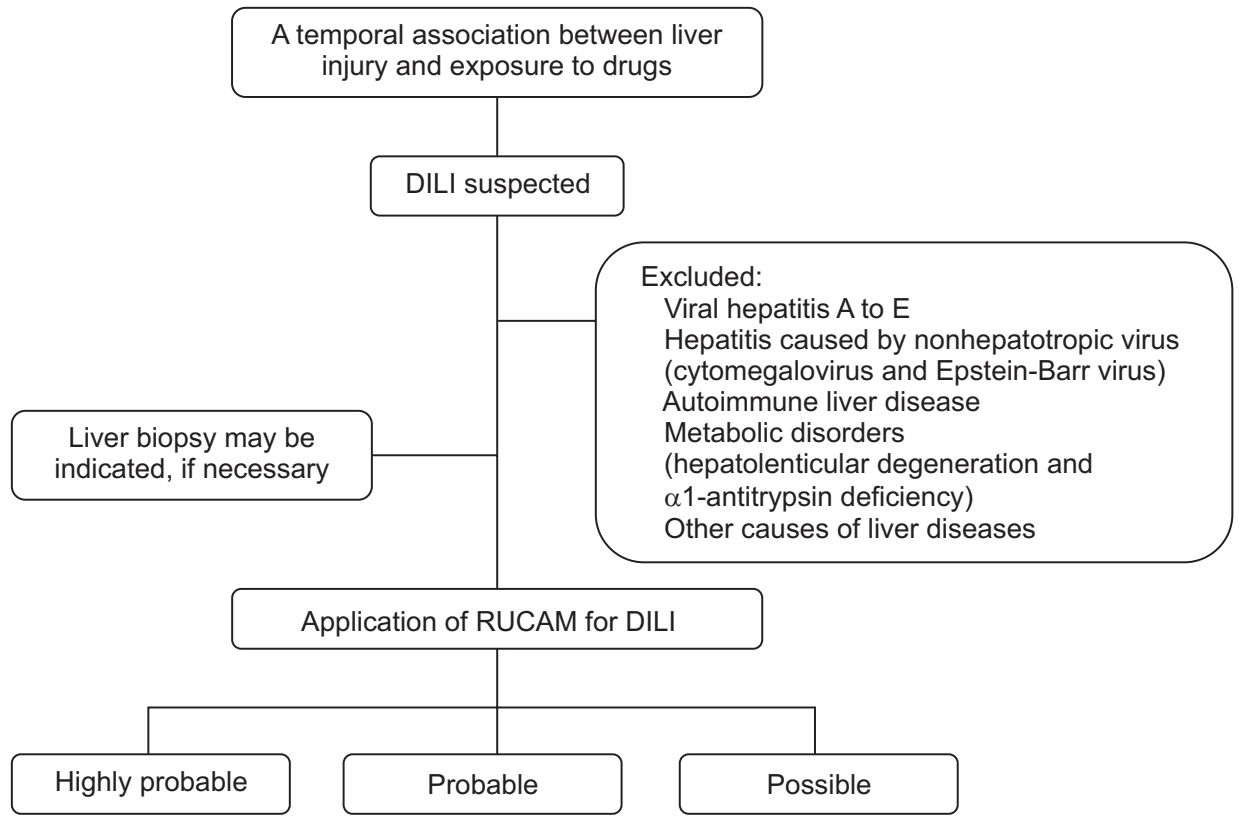

Fig. 1. Flowchart depicting the diagnosis of drug-induced liver injury (DILI) in children. RUCAM, Roussel Uclaf Causality Assessment Method. 
Table 1. Demographic, Clinical, Laboratory, and Pathological Characteristics of Children with Drug-Induced Liver Injury

\begin{tabular}{|c|c|c|c|}
\hline Characteristic & Value & Characteristic & Value \\
\hline Gender: boy & 66.7 & Clinical pattern of liver injury & \\
\hline Age, yr & $8(3,12)$ & Hepatocellular & 89.9 \\
\hline Medical history & & Cholestatic & 2.9 \\
\hline Respiratory infection & 37.7 & Mixed & 7.2 \\
\hline Fever of unknown origin & 15.9 & Laboratory variable & \\
\hline Tuberculosis* & 10.1 & Peak ALT, U/L & $649(215,1,125)$ \\
\hline Skin diseases & 7.2 & Peak AST, U/L & $434(145,968)$ \\
\hline Hematological disorders & 5.8 & Peak ALP, U/L & $287(224,419)$ \\
\hline Urinary diseases & 2.9 & Peak GGT, U/L & $100(39,176)$ \\
\hline Malignant tumor & 2.9 & Peak TB, mg/dL & $4.1(0.5,14.8)$ \\
\hline Ear, nose, and throat diseases & 2.9 & Peak TBA, $\mu \mathrm{mol} / \mathrm{L}$ & $93(12,342)$ \\
\hline Other $^{\dagger}$ & 14.6 & Lowest ALB, g/L & $37(34,40)$ \\
\hline Allergic history & 20.3 & Lowest CHE, U/L & $5,205(3,900,7,921)$ \\
\hline Clinical signs and symptom & & Peak PT, sec & $12.1(11.2,16.5)$ \\
\hline Jaundice & 59.4 & Lowest PA, \% & $85.0(51.8,97.5)$ \\
\hline Splenomegaly $^{\ddagger}$ & 47.8 & WBC count at baseline, $109 / \mathrm{L}^{\S}$ & $5.5(4.1,8.2)$ \\
\hline Gastrointestinal reaction & 44.9 & PLT count at baseline, $109 / \mathrm{L}^{\S}$ & $321(260,431)$ \\
\hline Hepatomegaly $^{\ddagger}$ & 36.2 & Pathological pattern of liver injury $(n=55)^{\|}$ & \\
\hline Fatigue & 34.8 & Acute hepatitis & 27.3 \\
\hline Fever & 31.9 & Chronic hepatitis & 47.3 \\
\hline Rash & 21.7 & Acute cholestasis & 3.6 \\
\hline Itching & 14.5 & Cholestatic hepatitis & 21.8 \\
\hline Arthralgia & 2.9 & & \\
\hline No symptoms & 20.3 & & \\
\hline
\end{tabular}

Data are presented as median (25th, 75th) or percent.

ALT, alanine transaminase; AST, aspartate transaminase; ALP, alkaline phosphatase; GGT, $\gamma$-glutamyltransferase; TB, total bilirubin; TBA, total biliary acid; ALB, albumin; CHE, cholinesterase; PT, prothrombin time; PA, prothrombin activity; WBC, white blood cell; PLT, platelet.

${ }^{*}$ One child exhibited the complication of epilepsy, and one developed nephrotic syndrome; ${ }^{\dagger}$ Six children received Chinese herbal medicine to improve symptoms. Two cases were caused by antibiotics, administered as prophylaxis against surgical infection; one was induced by montelukast for asthma; and one was caused by sotalol, administered for paroxysmal supraventricular tachycardia; ${ }^{\ddagger}$ Found by physical examination or

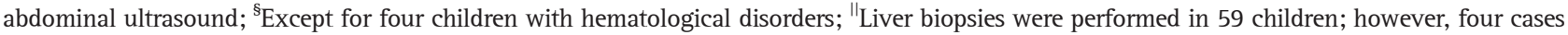
could not be classified into any pattern because of mild histological changes.

Mann-Whitney U test. Chi-square and Fisher exact tests were used to compare nominal variables. A p-value $<0.05$ (two-tailed) was considered statistically significant. All of the calculations were performed using SPSS version 16.0 software (SPSS Inc., Chicago, IL, USA).

\section{RESULTS}

\section{Clinical presentations}

Of 1,544 children hospitalized due to liver injury between January 2009 and December 2011, there were 69 children (4.5\%) with DILI. Demographic, clinical, and laboratory characteristics were shown in Table 1 . The median age of these cases was 8 years (range, 0.2 to 14.0 years). No child had pregnancy or alcohol or tobacco consumption. Among 65 children without hematological disorders, the white blood cell count at baseline was abnormally decreased in $83.1 \%$, and the platelet count was more than $300 \times 10^{9} / \mathrm{L}$ in $58.5 \%$ of the cases. Only one case had peripheral eosinophilia. Autoantibodies were detected in 32 children with DILI: antinuclear antibody was positive in four children and anti-liver-kidney microsome antibody was positive in one child.

\section{Implicated agents}

A list of agents implicated was provided in Table 2. Fifteen children (21.7\%) had been exposed to more than one type of drug before the liver injury occurred, with antibiotics commonly being combined with antipyretic analgesics and/or CHM for respiratory infection $(n=9)$ or fever of unknown origin (FUO) $(n=3)$. Four cases were caused by VitC-yin-qiao tablet consisting of acetaminophen, chlorpheniramine maleate, vitamin C, Lonicera japonica, Forsythia suspensa, Schizonepeta tenuifolia, 
Glycine max, Lophatherum gracile, Arctium lappa, Platycodon grandiflorum, Phragmites communis, Glycyrrhiza uralensis, and menthol. Among children in whom one type of drug was implicated, the major classes of WM causing DILI were as follows: antibiotics in 26.1\%, antituberculosis agents in 8.7\%, antipyretic analgesics in 5.8\%, antineoplastic agents in 5.8\%, and glucocorticoids in $4.3 \%$. Cephalosporin $(n=8)$ and macrolides $(n=6)$ were the most commonly implicated antibiotic agents. In 15 cases (21.7\%), CHM was implicated, and the details were shown in Table 3. The major implicated herbs were Ephedra sinica $(n=3)$ and Polygonum multiflorum $(\mathrm{n}=3)$. The characteristics of cases caused by CHM compared with WM were listed in Table 4. Of the six children with ALF, four were treated with CHM for tinea corporis $(n=1)$, vitiligo $(n=2)$, and poor appetite $(n=1)$, one was

Table 2. Implicated Agents in 69 Children with Drug-Induced Liver Injury

\begin{tabular}{lc}
\hline \multicolumn{1}{c}{ Implicated agent } & Value \\
\hline Combination of implicated drug & $15(21.7)$ \\
VitC-yin-qiao tablet* & 4 \\
Antibiotics+CHM+antipyretic analgesics & 3 \\
Antibiotics+CHM & 3 \\
Antibiotics+antipyretic analgesics & 2 \\
Antibiotics+antipyretic analgesics+antiviral agents & 1 \\
Antibiotics+CHM+antiparasitic agents & 1 \\
Antituberculosis agents+antiepileptic agents & 1 \\
Western medicine & $39(56.6)$ \\
Antibiotics & $18(26.1)$ \\
Cephalosporins (cephalexin, cefmetazole, & 8 \\
$\quad$ cefoperazone, ceftriaxone) & \\
Macrolides (azithromycin, roxithromycin) & 6 \\
Penicillins (amoxicillin) & 3 \\
Quinolones (norfloxacin) & 1 \\
Antituberculosis agents & $15(21.7)$ \\
Antineoplastic agents & 9 \\
Antipyretic analgesics & $6(8.7)$ \\
Glucocorticoids & $4(5.8)$ \\
Antiviral agents & $4(5.8)$ \\
Drug for asthma (montelukast) & $3(4.3)$ \\
Drug for paroxysmal supraventricular tachycardia & $2(2.9)$ \\
(sotalol) & $1(1.5)$ \\
ChM & $1.5)$ \\
Herbal decoction & \\
\hline & \\
\hline
\end{tabular}

Data are presented as number (\%).

CHM, Chinese herbal medicine.

*VitC-yin-qiao tablet consists of acetaminophen, chlorpheniramine maleate, vitamin C, Lonicera japonica, Forsythia suspensa, Schizonepeta tenuifolia, Glycine max, Lophatherum gracile, Arctium lappa, Platycodon grandiflorum, Phragmites communis, Glycyrrhiza uralensis, and menthol. caused by azithromycin for FUO, and another was induced by cephalosporins, antipyretic analgesics, and antiviral agents for pneumonia. Twenty-four chronic DILI cases were caused by antituberculosis agents (20.8\%), antineoplastic agents (16.7\%), antibiotics (16.7\%), combination of implicated drugs (16.7\%), glucocorticoids (12.5\%), CHM (8.3\%), and antipyretic analgesics (8.3\%), respectively.

\section{Pathological features}

Liver biopsies were performed in 59 children (85.5\%) and the pathological patterns of injury in 55 children were listed in Table 1, whereas four of the cases could not be classified into any pattern because of mild histological changes. Liver cell degeneration, necrosis, and lobular inflammation were common findings, with typical confluent necrosis in 11 cases (18.6\%), bridging necrosis in five cases (8.5\%), and submassive necrosis in one case who did not present with clinical features of liver failure. Twenty-nine biopsies (49.2\%) showed various degrees of interface hepatitis. Hepatocellular and/or canalicular cholestasis were observed in 16 cases (27.1\%). Twenty-nine biopsies (49.2\%) demonstrated eosinophil infiltration and none had duct sclerosis or loss. Typical examples of pathological patterns were described in Fig. 2. In 15 cases (25.4\%), the liver biopsy after the normalization of liver biochemistry still showed the histologic features of chronic hepatitis, such as moderate portal inflammation, interface hepatitis, fibroplastic proliferation, and fibrous septa formation. All three cases with ALF showed the pathological characteristics of cholestatic hepatitis involving confluent and bridging necrosis, cholestasis, interface hepatitis, and fibrous septa formation, but none demonstrated submassive necrosis.

\section{Outcomes}

There were two deaths caused by herbal decoctions for tinea corporis and poor appetite, respectively. Six cases presented with acute liver failure and 24 cases developed into chronic DILI. Fourteen children with a median TB level of $14.5(6.9,19.9) \mathrm{mg} /$ $\mathrm{dL}$ were treated with corticosteroids, of which seven developed chronic DILI, two presented with bacterial respiratory infection, two with oral cavity fungal infection, one with pulmonary fungal infection, and one with pyemia. In six children with ALF, two died, and the others recovered. The prognosis of the children with DILI caused by CHM and WM was shown in Table 4.

\section{DISCUSSION}

The diagnosis of DILI is challenging, especially in children, because there are no specific markers of DILI and the diagnosis largely depends on a high index of suspicion and the exclusion of other causes of liver diseases. ${ }^{10}$

DILI must always be considered, when there is a temporal association between observed liver injury and the exposure to 
Table 3. Chinese Herbal Medicine Used to Treat Drug-Induced Liver Injury in 15 Children

\begin{tabular}{|c|c|c|c|}
\hline Name & Aims of application & Classification & Potential herbals with hepatotoxicity \\
\hline \multicolumn{4}{|l|}{ Chinese patent medicine $(\mathrm{n}=9)$} \\
\hline Gan-mao soft capsule & URI & OTC & $\begin{array}{l}\text { Ephedra sinica Scutellaria baicalensis; } \\
\text { Mentha haplocalyx }\end{array}$ \\
\hline Xiao-er-ke-chuan-ling granule & URI & OTC & Ephedra sinica \\
\hline Dan-xiang-bi-yan tablet & Sinusitis & Px & Xanthium sibiricum Mentha haplocalyx \\
\hline Yan-hu-ning injection & Pneumonia & Px & $\begin{array}{l}\text { Andrographis paniculata } \\
\text { (dehydroandrographolide succinate) }\end{array}$ \\
\hline Xiao-er-kang granule & Poor appetite & Px & Unknown \\
\hline Shou-wu-yan-shou tablet & Vitiligo & OTC & Polygonum multiflorum \\
\hline Zang-qi-xue-yu capsule & Increasing energy levels & Not approved by CFDA & Agkistrodon halys pallas \\
\hline Xiao-er-pai-qian oral liquid & Hyperactivity & Not approved by CFDA & Smilax glabra \\
\hline Main-yi-wang capsule & Increasing energy levels & Not approved by CFDA & Unknown \\
\hline \multicolumn{4}{|l|}{ Herbal decoction $(\mathrm{n}=6)$} \\
\hline & URI & & Ephedra sinica \\
\hline & Vitiligo & & Polygonum multiflorum \\
\hline & Vitiligo & & Psoralea corylifolia \\
\hline & Tinea corporis & & Polygonum multiflorum \\
\hline & JRA & & Tripterygium wilfordii \\
\hline & Poor appetite & & Unknown \\
\hline
\end{tabular}

URI, upper respiratory infection; OTC, over-the-counter drug; Px, prescribed drug; CFDA, China Food and Drug Administration; JRA, juvenile rheumatoid arthritis.

drugs. The warning signal of liver injury has been either nonspecific symptoms (e.g., fatigue, nausea, vomiting, or jaundice) or, more commonly, biochemical dysfunction, which includes raised levels of ALT, ALP, or TB. Then viral (hepatitis A virus, hepatitis B virus, hepatitis C virus, hepatitis E virus, cytomegalovirus, and Epstein-Barr virus), autoimmune (antinuclear antibody and antismooth muscle antibody), and metabolic (Wilson's disease and $\alpha 1$-antitrypsin deficiency) disorders must be excluded. In some cases, liver biopsy may be indicated to exclude other diseases and to help make a diagnosis of DILI. ${ }^{10,22}$

The RUCAM is the most widely used for aiding in the causality assessment of DILI, but there have been several pitfalls in applying this method in children. ${ }^{23}$ No child has pregnancy or alcohol consumption, and therefore, no points are given in the risk factor components of age, alcohol, and pregnancy. In cases with multiple possible implicated agents, the combination of implicated drugs had been considered to be one pathogenic factor because it was technically difficult to identify which drug was the most likely cause of liver injury. ${ }^{22}$ CHM is less likely to be well characterized with regard to hepatotoxicity information, and this may compromise the RUCAM score for CHM because no points are given for herbs without existing information on hepatotoxicity. ${ }^{15}$ Therefore, the diagnostic test for DILI in children needs further investigation and validation.

The characteristics of DILI in children are different from those in adults due to age-related changes in drug metabolism and the special requirements of medication given to children. Although the data were from a single center, the results of this study might provide an opportunity to analyze the characteristics of DILI in children.

Similar to adult studies, a combination of implicated drugs is a major cause of DILI in children and antibiotics are the most commonly implicated drug class. ${ }^{16,24}$ In this study, antibiotics were commonly combined with antipyretic analgesics and/or CHM for FUO and respiratory infection, and the major implicated antibiotics were cephalosporin and macrolides. Compared with the DILIN prospective study, CHM is the main etiological agent of DILI in children in China because of the historical background of the use of CHM. ${ }^{8}$ In this study, the major implicated herbs were Ephedra sinica for respiratory infection and Polygonum multiflorum for skin diseases. The medical history of respiratory infection in $41.4 \%$ of cases is notable and may help the clinician identify children at risk.

Similar to adult, the most common pattern of DILI in children is hepatocellular injury, both in clinical (87.9\%) and pathological (76\%) categorization. ${ }^{16,21}$ The common presenting symptoms were fatigue, jaundice, and gastrointestinal reaction. However, this group of 58 cases in this study exhibited fewer hypersensitivity features (defined as fever, rash, and eosinophilia) than reported in pediatric DILI studies from Western countries and Indian. $^{8,17,25} 17.2 \%$ of the 58 cases had allergic history and 13.3\% of 30 cases had positive autoantibody, significantly lower than 
Table 4. Characteristics of Children with Drug-Induced Liver Injury Caused by Chinese Herbal Medicine Compared with Western Medicine

\begin{tabular}{|c|c|c|c|}
\hline Characteristic & Chinese herbal medicine $(n=15)$ & Western medicine $(n=39)$ & $\mathrm{p}$-value \\
\hline Age, yr & $10(4,12)$ & $7(3,12)$ & 0.438 \\
\hline Gender: boy & 60.0 & 71.8 & 0.403 \\
\hline Allergic history & 20.0 & 23.1 & 1.000 \\
\hline Days from drug start to symptoms & $30(7,90)$ & $10(3,30)$ & 0.048 \\
\hline \multicolumn{4}{|l|}{ Liver tests } \\
\hline Peak ALT, U/L & $649(349,1,010)$ & $529(186,1,038)$ & 0.569 \\
\hline Peak AST, U/L & $597(253,942)$ & $425(135,826)$ & 0.354 \\
\hline Peak ALP, U/L & $298(243,438)$ & $274(191,439)$ & 0.329 \\
\hline Peak GGT, U/L & $87(31,140)$ & $117(31,186)$ & 0.599 \\
\hline Peak TB, mg/dL & $16.6(3.7,22.2)$ & $1.4(0.4,10.1)$ & 0.004 \\
\hline Peak TBA, $\mu \mathrm{mol} / \mathrm{L}$ & $342(38,446)$ & $32(8,283)$ & 0.032 \\
\hline Lowest ALB, g/L & $37(31,40)$ & $38(35,39)$ & 0.394 \\
\hline Lowest CHE, U/L & $3,833(2,471,4,632)$ & $6,493(4,312,8,022)$ & 0.011 \\
\hline Peak PT, sec & $17.3(11.6,26.6)$ & $11.8(10.9,12.9)$ & 0.012 \\
\hline Lowest PA, \% & $45(31,89)$ & $90(74,102)$ & 0.006 \\
\hline \multicolumn{4}{|l|}{ Clinical pattern of liver injury } \\
\hline Hepatocellular & 100.0 & 87.2 & 0.347 \\
\hline Cholestatic & 0 & 2.6 & \\
\hline Mixed & 0 & 10.3 & \\
\hline \multicolumn{4}{|l|}{ Prognosis } \\
\hline Chronic & 13.3 & 46.2 & 0.031 \\
\hline ALF & 26.7 & 2.6 & 0.018 \\
\hline Death & 13.3 & 0 & 0.073 \\
\hline
\end{tabular}

Data are presented as median (25th, 75th) or percent.

ALT, alanine transaminase; AST, aspartate transaminase; ALP, alkaline phosphatase; GGT, $\gamma$-glutamyltransferase; TB, total bilirubin; TBA, total biliary acid; ALB, albumin; CHE, cholinesterase; PT, prothrombin time; PA, prothrombin activity; ALF, acute liver failure.

the $43 \%$ and $64 \%$ reported in the DILIN study, respectively. ${ }^{8}$ But our results were similar to other studies from China. In a similar study from China, 25.8\% of 31 children with DILI showed hypersensitivity features: itching in 3.2\%, fever in 6.5\%, rash in $3.2 \%$, and eosinophilia in $12.9 \%{ }^{6}$ Rash (16\%) and fever (14\%) were observed in 64 children with DILI in a single-center retrospective study from China. ${ }^{26}$ The difference in hypersensitivity features between China and Western countries needs further study and might be related to different classes of implicated agents and race-related genetic background.

Although most DILI resolves following drug discontinuation, up to $20 \%$ of patients progress to chronic DILI further challenging the clinicians diagnostic and management skills. ${ }^{27}$ This study revealed more frequent chronic DILI cases (25.9\%), defined as persistent biochemical abnormalities according to CIOMS, than reported in adult studies, which might be related to few cases with hypersensitivity features and the application of corticosteroids in this study. ${ }^{16,19,24}$ Compared to cases without hypersensitivity, children with hypersensitivity DILI present earlier, have less severe liver disease, and experience complete recovery. ${ }^{17}$ In this study, corticosteroids were used in 14 cases (24.1\%) with hyperbilirubinemia, 50\% of which developed chronic DILI and $43 \%$ presented with side effects of bacterial or fungal infection. However, it is likely that more severe and chronic cases of DILI were included in this study than are encountered in the general population, because all of the cases were hospitalized children in this study.

Several histologic features of DILI in children were illustrated in the present report. Nearly half of the cases were classified into chronic hepatitis in the pathological injury pattern, and $26.4 \%$ of liver biopsies after the normalization of liver tests still showed the pathological features of chronic hepatitis. Moreover, no case of ALF demonstrated submassive necrosis, and only one biopsy with submassive necrosis did not present the clinical features of liver failure. Thus, the correlation between the clinical pattern and the pathological categorization of injury is limited. ${ }^{21}$

Herbal medicine is widely used for treating diseases, improving symptoms, and overall health care, especially in China. Herbs have long been thought to be natural and safe. However, an increasing number of herbs and herbal products have been reported to cause liver injury. ${ }^{28,29}$ In this study, several characteristics of DILI in children caused by CHM were observed. Liver 


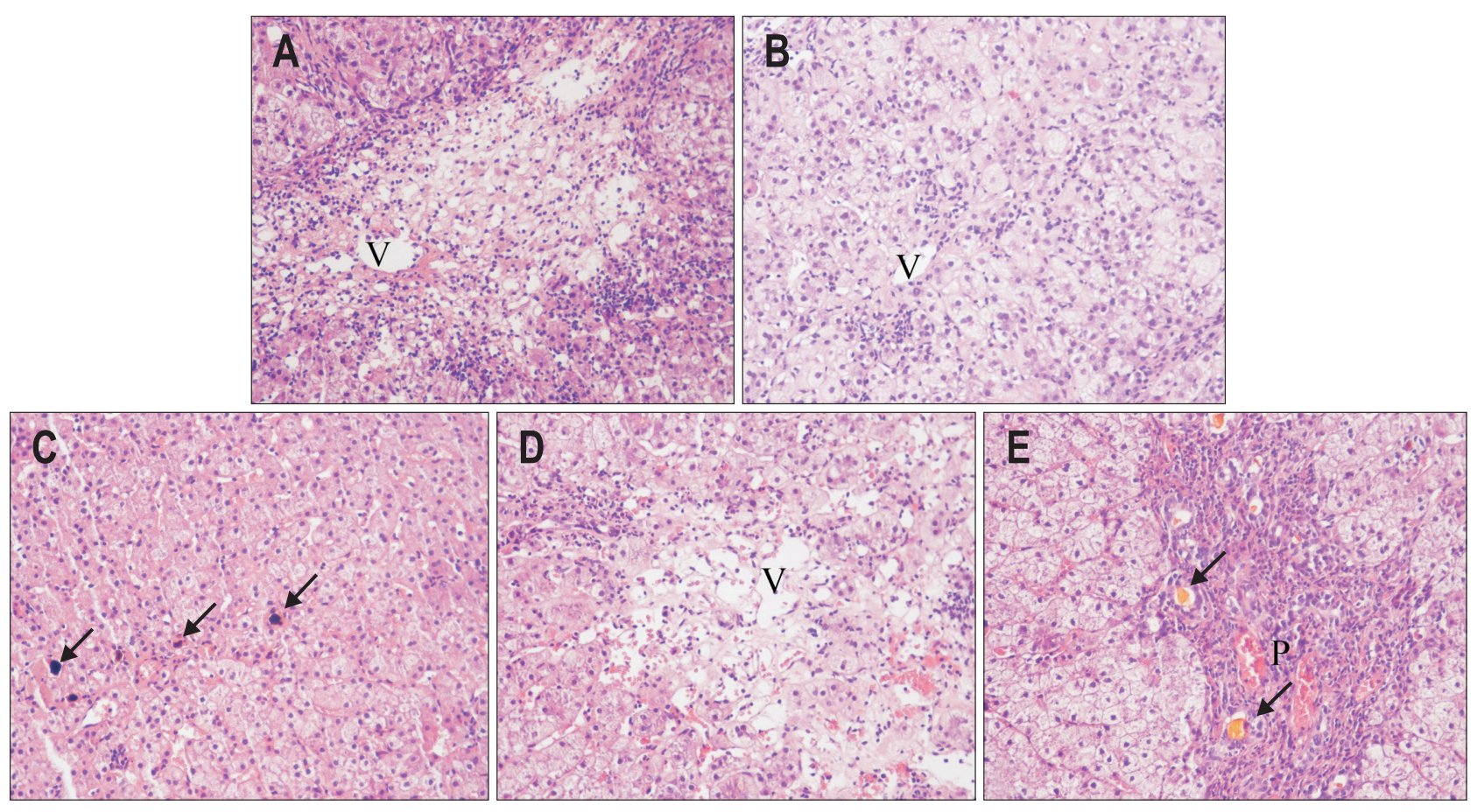

Fig. 2. Examples of the most common pathological injury patterns. (A) Acute hepatitis due to herbal decoction with Ephedra sinica for respiratory infection. Biopsy shows confluent and bridging necrosis around the central vein and significant lobular inflammation. (B) Chronic hepatitis due to the combination of cephalosporin antibiotics and antipyretic analgesics for fever of unknown origin. Liver biopsy shows fibrous septa formation and moderate interface hepatitis. (C) Acute cholestasis due to azithromycin. Biopsy showed hepatocellular and canalicular cholestasis with bile plugs. (D, E) Cholestatic hepatitis due to herbal decoction with Polygonum multiflorum for vitiligo. Biopsy showed prominent canalicular cholestasis, confluent necrosis, and neutrophilic infiltration (H\&E stain, $\times 200$; for orientation, V indicates the central vein, P indicates the portal area, and arrows indicate cholestasis).

injury caused by CHM was hepatocellular, the severity ranged from mild injury to ALF, and the major implicated herbs were Ephedra sinica for respiratory infection and Polygonum multiflorum for skin diseases. Numerous herbs have been described to cause hepatotoxicity, including Ephedra sinica, Polygonum multiflorum, Mentha haplocalyx, Scutellaria baicalensis, Xanthium sibiricum, Andrographis paniculata (dehydroandrographolide succinate), Agkistrodon halys pallas, Smilax glabra, Tripterygium wilfordii, and Psoralea corylifolia. ${ }^{15,30-33}$

However, it is extremely difficult to identify the exact causative hepatotoxic compounds and the mechanisms of their hepatotoxicity, because CHM consists of multiple herbs or constituents and there are herb-drug or herb-herb interactions leading to potentiation of risk for hepatotoxicity. ${ }^{15,28}$ Many herbals, such as Glycyrrhiza uralensis, Arctium lappa, Glycine max, Pueraria lobota, and Atractylodes macrocephala, have been identified as substrates, inhibitors, or inducers of cytochromes P450. 15,34,35 Glycyrrhiza uralensis, Arctium lappa, and Glycine max are constituents of VitC-yin-qiao tablet, and whether these herbs might potentiate the intrinsic hepatotoxicity of acetaminophen requires further study.

In this study, the cases caused by CHM showed more severe liver injury. Three cases with ALF and only one death were caused by CHM. Compared with WM, the cases with DILI caused by CHM have higher median levels of TB and total biliary acid, longer PT, lower prothrombin activity and lower cholinesterase (all $\mathrm{p}<0.05$ ). According to Hy's law, jaundice is a good predictor of mortality in DILI. ${ }^{36}$ In general, the higher the serum bilirubin, the more severe the liver injury. ${ }^{37}$ According to a research from multicenters in China, CHM was also reported be the main etiological agent of acute severe DILI and the cure rate of acute severe DILI was low (6.6\%). ${ }^{14}$ However, $13.3 \%$ of the cases caused by CHM were associated with chronic DILI, less than that caused by WM $(46.2 \%)(p=0.031)$. CHM is likely to cause severe liver injury and even death, but the liver injury caused by CHM could be curable after stopping causative CHM and being treated. In the chronic DILI cases caused by WM, some needed continue treatment of primary diseases, such as tuberculosis, malignant tumor, and hematological disorders. Prolonged medication administration and drug rechallenge might be risk factors for developing chronic DILI. ${ }^{38}$ Antituberculosis agents, antineoplastic agents, antibiotics, glucocorticoids, and antipyretic analgesics have been reported to cause chronic DILI. ${ }^{39,40}$ In the multicenter prospective study, malignancy receiving antineoplastic agents might be a risk factor for developing chronic DILI. ${ }^{40}$ However, there was selection bias because all of the cases were hospitalized and it could result in more chronic DILI cases. In additional, the children with DILI caused by CHM had a greater median 
number of days from drug start to symptoms than WM, but this trend was of borderline statistical significance $(\mathrm{p}=0.048)$.

Notably, the preparation of some CHM has not been provided and even some Chinese patent medicines have not been approved by China Food and Drug Administration. The dosage and course of treatment for children have not been provided in the instructions for most Chinese patent medicines. Thus, studies are strongly needed to improve CHM safety in children.

Although this study, which reported the largest number of children with DILI, was one of the few efforts to investigate DILI in children, it was a single-center and retrospective survey and was limited by potential selection bias because all of the cases were hospitalized children, leading to a poor outcome and low presentation in the general population.

In summary, the clinical characteristics of pediatric DILI are diverse, ranging from asymptomatic hepatitis to acute liver failure, and both chronicity and mortality are observed. Most of children with DILI typically present with hepatocellular injury pattern. It is important for pediatricians to evaluate the potential hepatotoxicity of commonly used CHM or antibiotics, especially the combination of these drugs, for respiratory infection, and monitor children with DILI during the recovery phase because of the slow pathological repair of liver injury after the normalization of liver biochemistry. Pediatricians should pay great attention to herbal hepatotoxicity and take measures to prevent development of severe liver injury induced by CHM. DILI is an important and problematic cause of liver injury in children, and further efforts are needed to study the mechanisms, risk factors, and outcomes of pediatric DILI and to develop methods for its diagnosis, prevention, and treatment.

\section{CONFLICTS OF INTEREST}

No potential conflict of interest relevant to this article was reported.

\section{ACKNOWLEDGEMENTS}

This work was funded by the National Natural Science Foundation of China (number: 81303120) and the National Key Technology R\&D Program (number: 2012BAI29B02).

\section{REFERENCES}

1. Zhu XX, Zhu Y, Wan CM. Clinical features of drug-induced liver injury in children. Zhongguo Dang Dai Er Ke Za Zhi 2012;14:131133.

2. Squires RH Jr, Shneider BL, Bucuvalas J, et al. Acute liver failure in children: the first 348 patients in the pediatric acute liver failure study group. J Pediatr 2006;148:652-658.

3. Murray KF, Hadzic N, Wirth S, Bassett M, Kelly D. Drug-related hepatotoxicity and acute liver failure. J Pediatr Gastroenterol Nutr
2008;47:395-405.

4. Orostegui L, Medejel N, Kone-Paut I, Dreyfus M, Benhamou D. Fondaparinux (Arixtra*) hepatotoxicity in a 6 year-old child. J Hepatol 2013;58:195-198.

5. Okada T, Honda S, Miyagi H, Minato M, Kubota KC, Taketomi A. Nafamostat mesilate-induced hepatocellular necrosis in a child with pancreatitis caused by pancreaticobiliary maljunction: druginduced liver injury. Pancreas 2012;41:981-983.

6. Wang SZ, Gao S, Liu YM, et al. Clinical characteristics of druginduced liver injury in 31 pediatric cases. Zhonghua Gan Zang Bing Za Zhi 2012;20:193-195.

7. Hita EO, García JA, Gonzalez JC, et al. Amoxicillin-clavulanic acid hepatotoxicity in children. J Pediatr Gastroenterol Nutr 2012;55: 663-667.

8. Molleston JP, Fontana RJ, Lopez MJ, et al. Characteristics of idiosyncratic drug-induced liver injury in children: results from the DILIN prospective study. J Pediatr Gastroenterol Nutr 2011;53: 182-189.

9. Johnson TN, Thomson M. Intestinal metabolism and transport of drugs in children: the effects of age and disease. J Pediatr Gastroenterol Nutr 2008;47:3-10.

10. Piñeiro-Carrero VM, Piñeiro EO. Liver. Pediatrics 2004;113(4 Suppl):1097-1106.

11. Faa G, Ekstrom J, Castagnola M, Gibo Y, Ottonello G, Fanos V. A developmental approach to drug-induced liver injury in newborns and children. Curr Med Chem 2012;19:4581-4594.

12. Fontana RJ, Seeff LB, Andrade RJ, et al. Standardization of nomenclature and causality assessment in drug-induced liver injury: summary of a clinical research workshop. Hepatology 2010;52: 730-742.

13. Wu CH, Wang CC, Kennedy J. The prevalence of herb and dietary supplement use among children and adolescents in the United States: results from the 2007 National Health Interview Survey. Complement Ther Med 2013;21:358-363.

14. Hepatobiliary Collaborative Group, Chinese Society of Gastroenterology. National multi-centers survey on inpatients with acute severe drug-induced liver injury. Chin J Dig 2013;33:80-83.

15. Bunchorntavakul C, Reddy KR. Review article: herbal and dietary supplement hepatotoxicity. Aliment Pharmacol Ther 2013;37:317.

16. Chalasani N, Fontana RJ, Bonkovsky HL, et al. Causes, clinical features, and outcomes from a prospective study of drug-induced liver injury in the United States. Gastroenterology 2008;135:19241934.e4.

17. Devarbhavi H, Karanth D, Prasanna KS, Adarsh CK, Patil M. DrugInduced liver injury with hypersensitivity features has a better outcome: a single-center experience of 39 children and adolescents. Hepatology 2011;54:1344-1350.

18. Danan G, Benichou C. Causality assessment of adverse reactions to drugs: I. a novel method based on the conclusions of international consensus meetings: application to drug-induced liver injuries. J Clin Epidemiol 1993;46:1323-1330. 
19. Bénichou C. Criteria of drug-induced liver disorders: report of an international consensus meeting. J Hepatol 1990;11:272-276.

20. Kleiner DE. The pathology of drug-induced liver injury. Semin Liver Dis 2009;29:364-372.

21. Kleiner DE, Chalasani NP, Lee WM, et al. Hepatic histological findings in suspected drug-induced liver injury: systematic evaluation and clinical associations. Hepatology 2014;59:661-670.

22. Rockey DC, Seeff LB, Rochon J, et al. Causality assessment in drug-induced liver injury using a structured expert opinion process: comparison to the Roussel-Uclaf causality assessment method. Hepatology 2010;51:2117-2126.

23. Rochon J, Protiva P, Seeff LB, et al. Reliability of the Roussel Uclaf Causality Assessment Method for assessing causality in druginduced liver injury. Hepatology 2008;48:1175-1183.

24. Andrade RJ, Lucena MI, Fernández MC, et al. Drug-induced liver injury: an analysis of 461 incidences submitted to the Spanish registry over a 10-year period. Gastroenterology 2005;129:512521.

25. Hunt CM. Mitochondrial and immunoallergic injury increase risk of positive drug rechallenge after drug-induced liver injury: a systematic review. Hepatology 2010;52:2216-2222.

26. Ding QY, Yu HY, Sun WW, Pan J, Cao XG, Wang L. The clinical analysis of drug induced hepatitis in child. Jiangsu Med J 2010; 36:339-340.

27. Hayashi PH, Fontana RJ. Clinical features, diagnosis, and natural history of drug-induced liver injury. Semin Liver Dis 2014;34:134144.

28. Schoepfer AM, Engel A, Fattinger K, et al. Herbal does not mean innocuous: ten cases of severe hepatotoxicity associated with dietary supplements from Herbalife products. J Hepatol 2007;47: 521-526.
29. Li FY, Li J, Xiao XH. Research status of herbal drug-induced liver injury. Chin J Tradit Chin Med Pharmacol 2009;24:265-269.

30. Skoulidis F, Alexander GJ, Davies SE. Ma huang associated acute liver failure requiring liver transplantation. Eur J Gastroenterol Hepatol 2005;17:581-584.

31. Liu HC, Liu HY. The liver damage of herbs and the other botanical herbs. Chin Hepatol 2001;6:52-53.

32. Liu SM. Herbal drug-induced liver injury. Beijing: China Press of Traditional Chinese Medicine, 2007:68-70, 248-250, 278-280.

33. Nam SW, Baek JT, Lee DS, Kang SB, Ahn BM, Chung KW. A case of acute cholestatic hepatitis associated with the seeds of Psoralea corylifolia (Boh-Gol-Zhee). Clin Toxicol (Phila) 2005;43:589-591.

34. Dong Y, Wang J, Yang Q, Wang YW, Zhu XX. Current status on the interaction of cytochrome P450 and metabolism of Chinese materia medica. Chin J Inf Tradit Chin Med 2011;18:100-103.

35. Guerra MC, Speroni E, Broccoli M, et al. Comparison between chinese medical herb Pueraria lobata crude extract and its main isoflavone puerarin antioxidant properties and effects on rat liver CYP-catalysed drug metabolism. Life Sci 2000;67:2997-3006.

36. Björnsson E. Drug-induced liver injury: Hy's rule revisited. Clin Pharmacol Ther 2006;79:521-528.

37. Verma S, Kaplowitz N. Diagnosis, management and prevention of drug-induced liver injury. Gut 2009;58:1555-1564.

38. Aithal PG, Day CP. The natural history of histologically proved drug induced liver disease. Gut 1999;44:731-735.

39. Rangnekar AS, Fontana RJ. An update on drug induced liver injury. Minerva Gastroenterol Dietol 2011;57:213-229.

40. Fontana RJ, Hayashi PH, Gu J, et al. Idiosyncratic drug-induced liver injury is associated with substantial morbidity and mortality within 6 months from onset. Gastroenterology 2014;147:96-108. e4. 